\title{
INTEGRATED INJECTION LOGIC
}

\author{
By \\ Mark Grabosky \\ 5th Year Microelectronics Student \\ Rochester Institute of Technology
}

\section{ABSTRACT}

Since Integrated Injection Logic was developed in 1972, it has found applications in LSI memories, microprocessors, digital wrist watches, $A / D$ and $D / A$ converters, and other custom IC's. The characteristics of a typical IIL gate are comparable to those of CMOS devices. Propagation times as low as $63 \mathrm{~ns}$ and power consumptions as small as $.01 \mathrm{~mW}$ per gate have been achieved in well developed IIL processes.

The major benefit that IIL has over other logic families is its relative simplicity. A standard IIL process only requires four masks, (base diffusion, emitter diffusion, contact cuts, and metallization). No isolation is required for an IIL process thus no epitaxial layer is needed nor is ion implantation or localized oxidation.

II gates were processed as part of a double diffused bipolar project. Functional tests were performed on NOR and AND gates as to examine aspects of the design and processing. Suggestions for improving device characteristics for future IIL designs are also given.

\section{INTRODUCTION}

Integräted Injection Logic is much like Direct Coupled Transistor Logic. In appendix A, Fig. 4.13-1 thru 4.13-4 show the evolution of IIL circuits from DCTL. The major advantage of using IIL over DCTL is that IIL makes use of a merged transistor design that saves real estate on the chip. Also the relative simplicity in circuit design makes the layout of an IIL gate very easy. From figure 4.13-3 to figure 4.13-4 in Appendix A note that the pull up resistors Rb have been replaced with PNP transistors with common emitters and bases. This is done to reduce the large space required to fabricate the resistors on a chip.

Because the circuit design calls for a PNP transistor to be connected to an NPN transistor and since the base of the PNP is connected to the emitter of the NPN and the collector of PNP to the base of the NPN, the two transistors may be merged together and share like diffusions as illustrated in figure $A$.

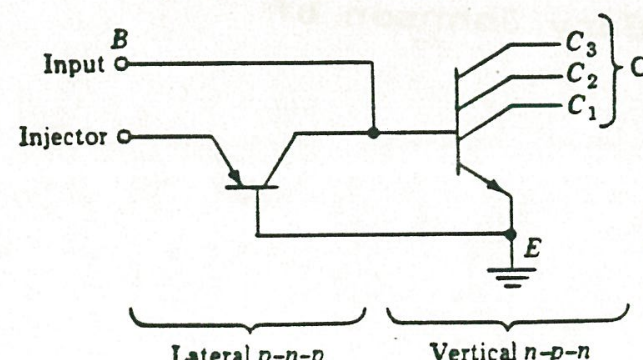

Lateral $p-n-p \quad$ Vertical $n-p-n$

Figure $A$
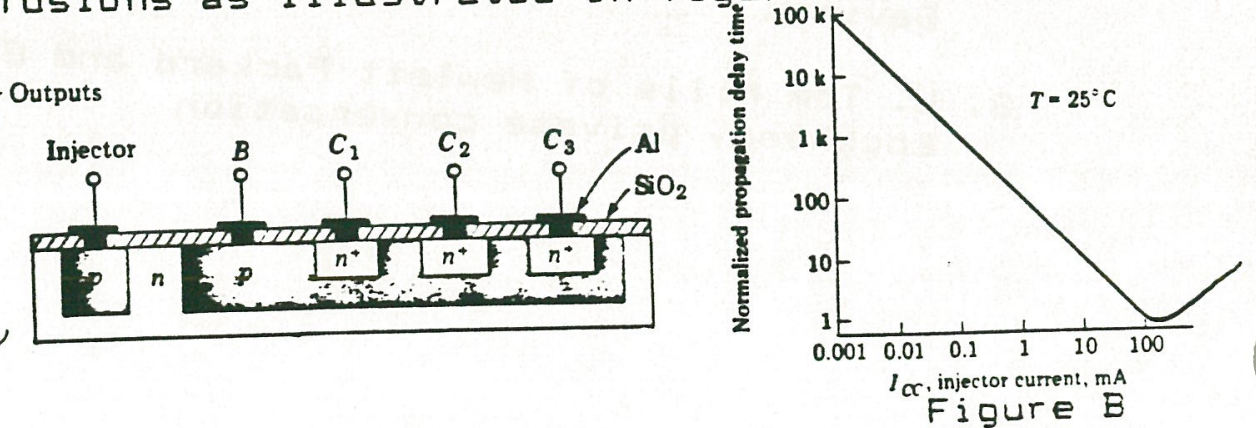
The emitter of the lateral PNP is referred to as the injector. It is biased thru an external resistor, $R_{I N J}$ to a positive supply voltage. By adjusting the value of propagation times can be made faster at propagation delay time as a consumption. Figure $B$ shows a plot of the Texas Instrument SBPO400 function of the injector current for the

THEORY

To have a successful IIL process for LSI circuits where a large number of gates will gain of at least one. Consider the for each to have a cur
circuit in figure $c$.

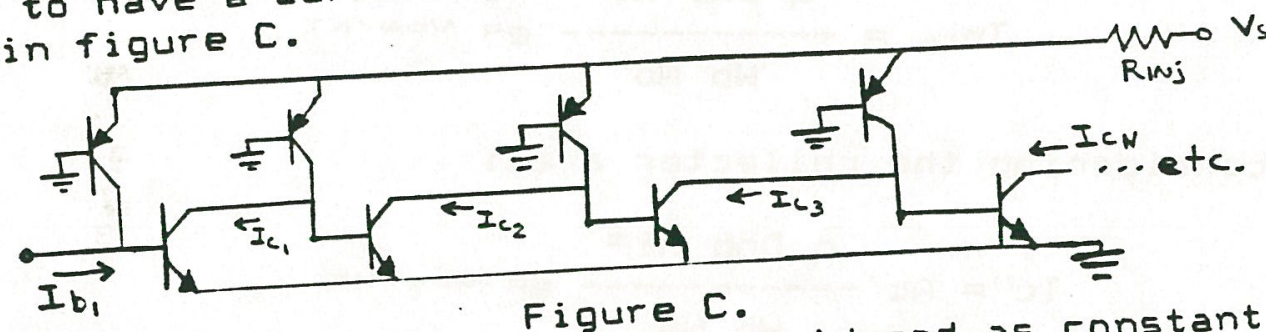

Figure C. If the PNP lateral transistors are considerexpressed as: sources then the small signal

$$
\text { Gain }=\frac{I C_{N}}{I b_{1}}=- \text { Betan }^{N}
$$

Thus if Beta is less than one, the small signal gain of the chain will be degraded with each stage until it cannot be distinguis on from the background noise. If Beta is greatem a high state of Vbe digital operation of the circuit wi the number of stages in the to a low of-vee sat, regardless of thansistors in an II circuit circuit. However, the NPN vertical gains are hard to come by. operate in reverse mode, 50 high gaverse mode NPN vertical

Let's examine the gain found from the minority carrier transistor. The gain cancentrations under active biasing.

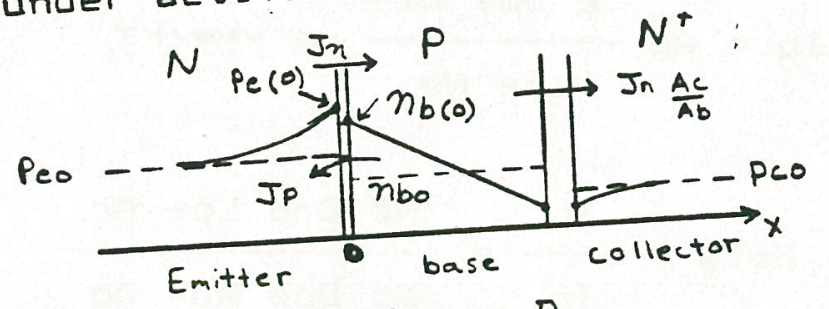

Figure $D$. emitter into the base can

urrent injected from the

$$
\begin{aligned}
& \text { The expressed as: } \\
& \text { be. } \quad \text { In }=a \text { Dnb } \frac{d}{d x}(n b-n b 0)
\end{aligned}
$$

Similarly, the hole current flowing from the base into the emitter can be expressed as:
$3 a . \quad$ Jp $=$ a Dpe $\frac{d x}{d}-(p e-p e 0)$ 
In the regions where the base width is short, (the width of the base is less than one diffusion length), electrons injected from the emitter into the base will diffuse across the base with no recombination. The electrons are quickly swept away at the basecollector junction by the reverse biased field. Thus the concentration gradient in the base will be linear and since nbo is many orders of magnitude less than nb(0) under bias:

2b.

$$
\frac{d}{d x}(n b-n b 0)=\frac{n b(0)}{w b}=\frac{n b 0}{w b} e^{a} \text { vocrkt }
$$

Where $n b O=n i=/ N b$

Thus:

$2 c$.

$$
I n_{-b}=\frac{\text { a Dnb } n i z}{\text { Wb } N b}
$$

And considering the collector area:

$2 d$.

$$
\text { Ic }=A C \frac{\text { a Dnb } n i e}{\text { Wb } N b}
$$

For the hole current in the emitter, since the width of the emitter is greater than the diffusion length, (LPE), the charge gradient at the emitter-base junction can be shown as:

3b.

$$
\frac{d x}{d}-(p e-p e 0)=\frac{\text { Lpe }}{\text { pe }(0)}=\frac{\text { Lpe }}{\text { peo }}
$$

Where peO $=n i$ The $/ \mathrm{Ne}$

Thus:

$3 c$.

$$
J_{p_{b-}}=\frac{\text { q Dpe } n_{i=}}{\text { Lpe } \mathrm{Ne}}
$$

And considering the base area:

$3 d$.

$$
\text { प Dpe nie }
$$

LPE Ne

And

$$
\text { 4. Beta }=\frac{\text { Ic }}{\text { Ib }}=\frac{\text { Ne Dnb Lpe } A c}{\text { Nb Dpe Wb } \mathrm{Ab}}
$$

By examining equation 4 one can manipulate the processing conditions to get a good gain. However, since the vertical transistors are operating in reverse mode, the substrate is the emitter and the doping profile will look something like figure E.

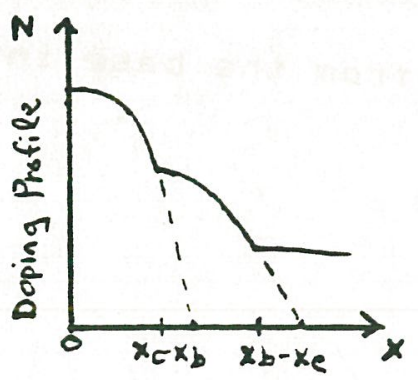

Figure $E$

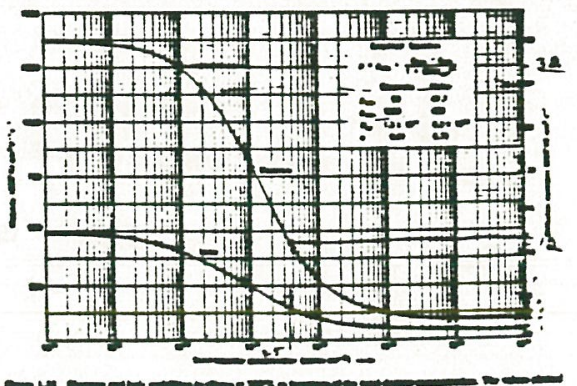

Figure $F$ 
Thus, as a consequence of the geometries, Nb must be greater than
However, this should be made as $\mathrm{Ne}$ and $\mathrm{Ne} / \mathrm{Nb}$ is less than one. However, this low boron

close to one as possible. Noter a long drive-in and subsequent concentration is required, afte base region may become depleted of oxidations, the surface in the base by doing a double boron boron. This problem may be avoided bive-in, the wafer may be diffusion. After doing the base drivend, very short, predeposit remasked with the base mask and aceeding with the process. of boron may be done before Proce/DPe from figure F, shows that the Looking at the ratiogable. For $\mathrm{Ne}=2.0 \mathrm{E} 15, \mathrm{Nb}=1.0 \mathrm{O} 16$ : diffusivity ratio is favorable.

5. $\quad \frac{N e}{N b}=0.2 \quad \frac{D n b}{D p}=\frac{32}{12}=2.67$ LPe is determined by the emitter doping concentration. For
Ne $=2.0 E 15$. The diffusion length LPe is:

6.

$$
L P_{-}=20 \mathrm{um}
$$

How small can we make the base width? The base width is limited by the punch-thru voltage. Punch thru-occurs when the depletion width of the B-C junction extends follows. width of the base. It can

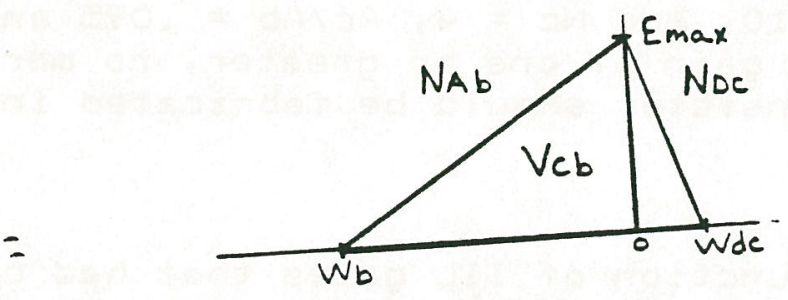

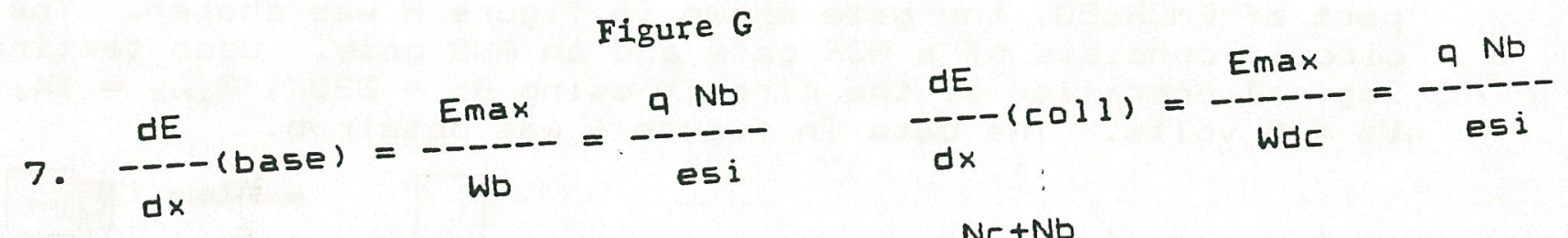

NC+Nb

B.

$$
W_{D \rightarrow D 1-t i k a n}=W_{b}+W d C=\frac{N E}{N E}
$$

9.

$$
\text { Vce }=.5 \text { Emax WDmpizaticion }
$$

Thus combining eq. 7,8 , and 9:

10.

$$
w_{b} \min =\frac{2 \text { Veed esi Ne }}{\text { q Nb(NC+Nb) }}
$$
For a lov source wh min. will be 1.08 m. The maximum gain from
equation 4 using the values from 5,6 , and 10 , will be:

11. Beta $=\frac{(1)(32)(20) \mathrm{AC}}{(5)(12)(01) \mathrm{Ab}}=10.67$ 


\section{DESIGN RULES}

When designing the physical layout of an IIL gate, one should obey the following ground rules.

1. All collectors should be square.

2. The space between collectors should be Md.

3. The width of the base diffusions should be Wc + 2Md.

4. The contacts to the injector rail should be at the end of the injector diffusions so that the base diffusions need not extend beneath the injector metallization.

5. Injector rails should be placed between each base diffusion.

6. The emitter/substrate contact should be N+ doped and should be made large as to minimize its resistance.

Where: $W_{c}=$ Topographical collector width

Md = Minimum dimension allowed

If the Ground Rules are obeyed, the ratio of areas may be found from:

$$
A c / A b=\frac{W c e}{\left(W_{C}+2 M d\right)\left[N C\left(W_{C}+M d\right)+W c\right]}
$$

For $W c=30, M d=10$, and $N c=4, A c / A b=.095$ and Beta $=1.01$. Therefore to get a gain of one or greater, no more than four collectors per transistor should be fabricated in this process.

\section{EXPERIMENT =}

To test the function of IIL gates that had been fabricated as part of EMCR650, the gate shown in figure $H$ was chosen. The circuit consists of a NDR gate and an AND gate. Upon testing the logical operation of the circuit using $R O=330 K, R_{I N J}=1 K$, and $v_{5}=5$ volts. The data in figure 6 was obtained.

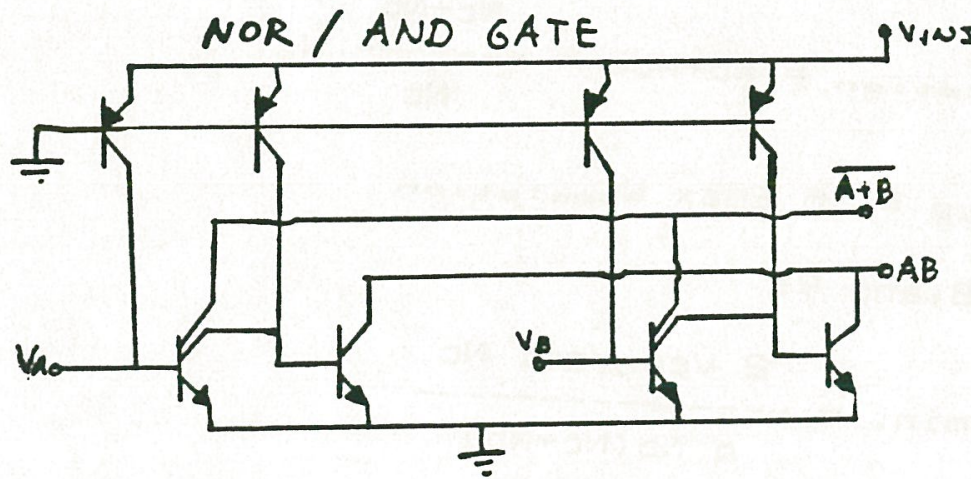

Figure H

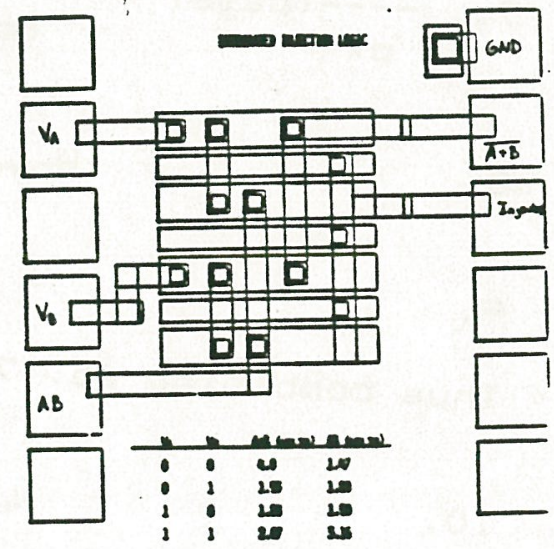

His

\section{RESULTS AND DISCUSSION}

The on voltage of the NOR gate was measured to be $4.0 \mathrm{~V}$. This drop of one volt across $R$ out when the transistor should be 
off is due to the NPN transistor leakage current. The higher than expected output of about two volts observed when the output should be low is caused by an internal emitter resistance of 550 ohms. (see appendix $B$ ) This was determined by examining Vee at $I c=0$. At $V c e=0$, dVce/dIb $=550$ OHMS. Notice that the collector current is negative until the collector voltage becomes greater than the drop across the internal emitter resistance. This is a big problem and should try to be avoided by making the emitter contact big. N+ doped, close to the gates, and sintering well. With this emitter resistance present, one can never achieve a low state of zero volts. Also the leakage current is quite significant when a supply voltage of five volts is used because of the low breakdown voltage, VceO $=6$ volts. The breakdown voltage is low due to the high doping concentration of the base.

The gain of the reverse mode NPN was only .08. So the logical voltage swing is degraded with each stage. This is seen from the voltage swing of the AND gate which is lower than the swing of the NOR gate because the AND gate is a two stage device while the NOR gate a one stage device.

\section{CONCLUSION}

The reason why the gain is 50 low in this circuit is that the doping of the base in those devices was high, about 5.0 E16, and $A c / A b=.067$. Appendix $C$ shows the doping profiles as modeled by SUPREM and the gain calculations. Please note that this gate was processed along with discrete transistors with goal of obtaining high forward gains. (Appendix D)

However, the results are encouraging. Although the voltage swings, from logic one to 109 ic zero, are small, they are definitely present and in correlation with the expected operation of these ga'tes. If the IIL gates are more carefully designed and the processing is performed to get high reverse mode gains, functional LSI IIL gates should be no problem.

\section{REFERENCES}

1. Jacob Milliman: "Microelectronics," Chapter 9, MeGraw-Hill Book Company, New York, 1979

2. Herbert Taub, Donald Schilling: "Digital Integrated Electronic5," Chapter 4, McGraw-Hili Book Company, New York, 1977 3. S.M. Sze: "VLSI Technology," Chapter 11, MeGraw-Hill Book
Company, New York, 1983

\section{ACKNOWLEDGEMENTS}

J. Lekas: His circuit design used for testing is shown in figure $\theta$

R. Turkman: The affect of AC/Ab on the reverse mode current gain.

P. George: For helping me put this report together. 


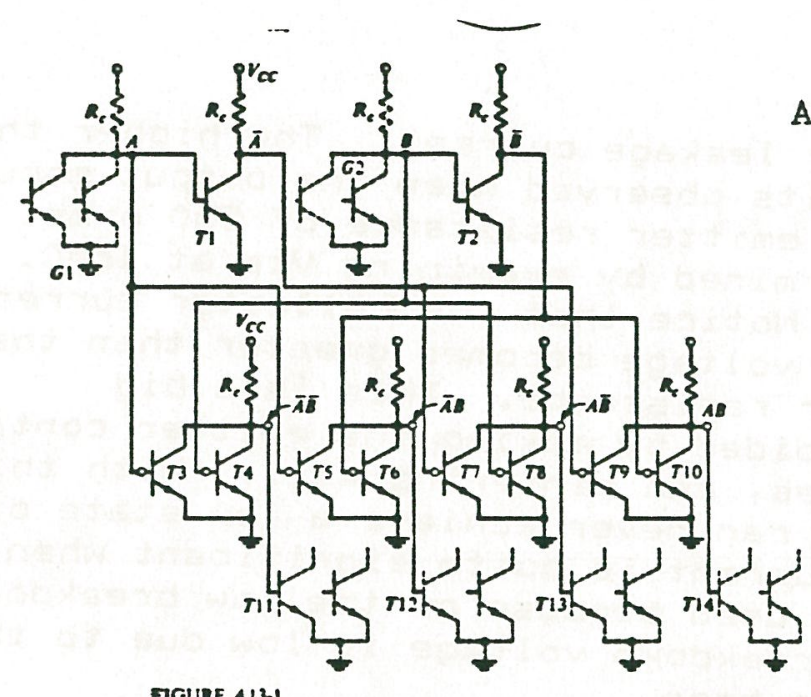

FGURE 4.13-1

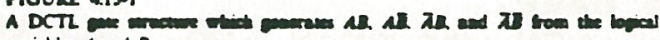
A Dieble 140.

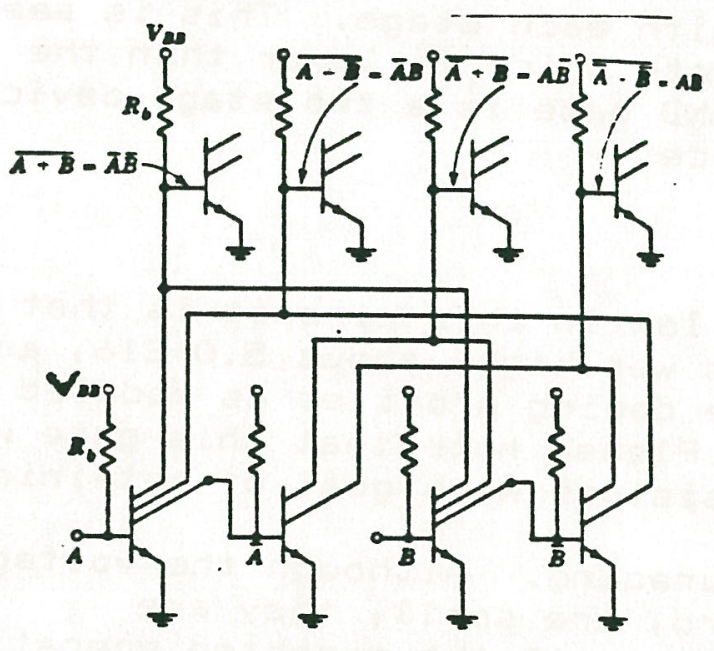

FIGURE 4.13.5-

Figure 4.13-2 redrawe with enleiple-collector transistors.
APPENDIX A

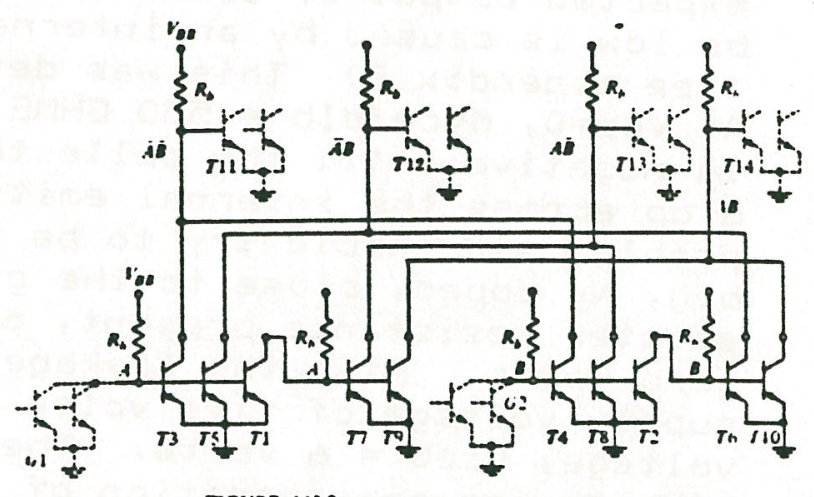

FGURE 4.13-2

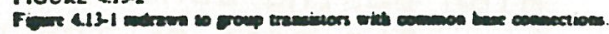

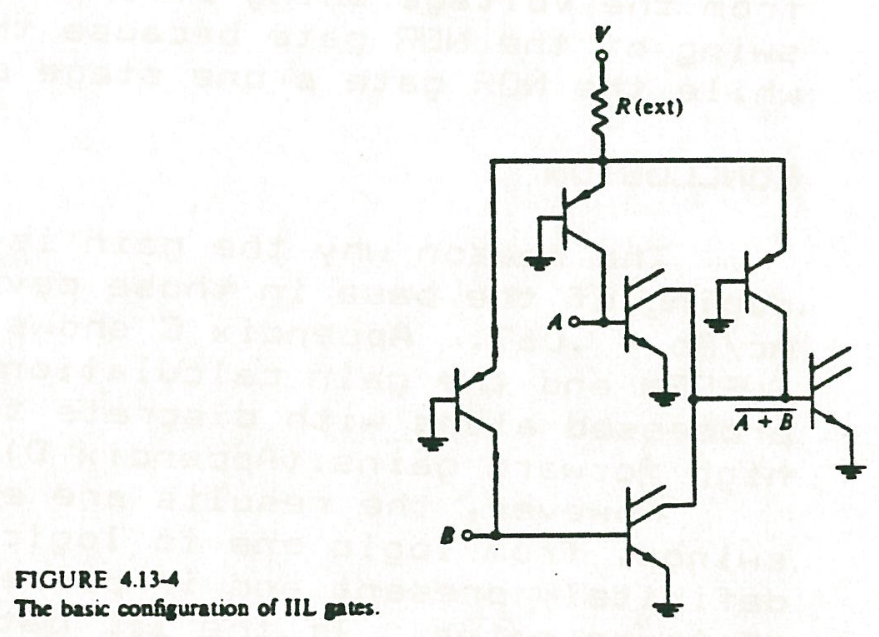

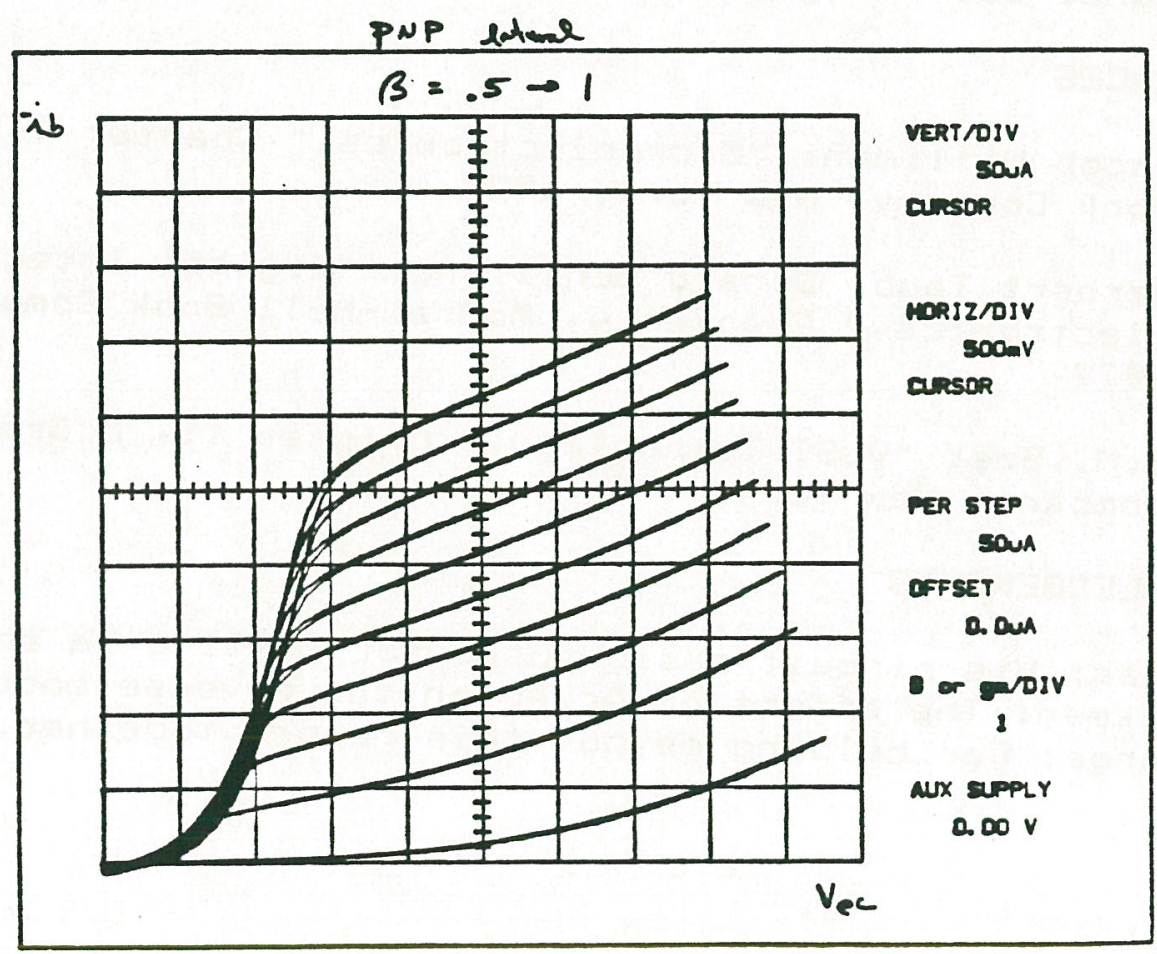




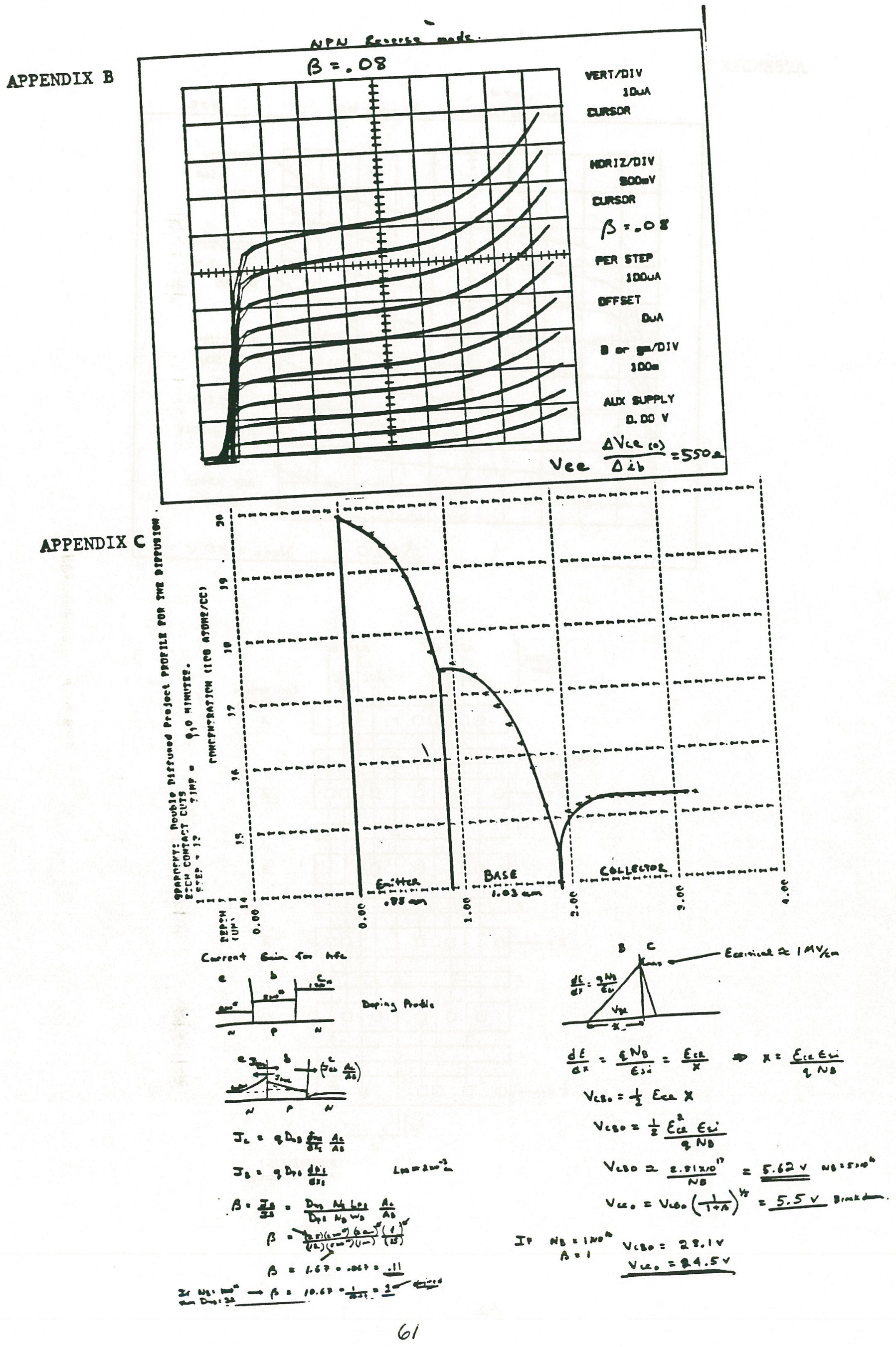


APPENDIX D
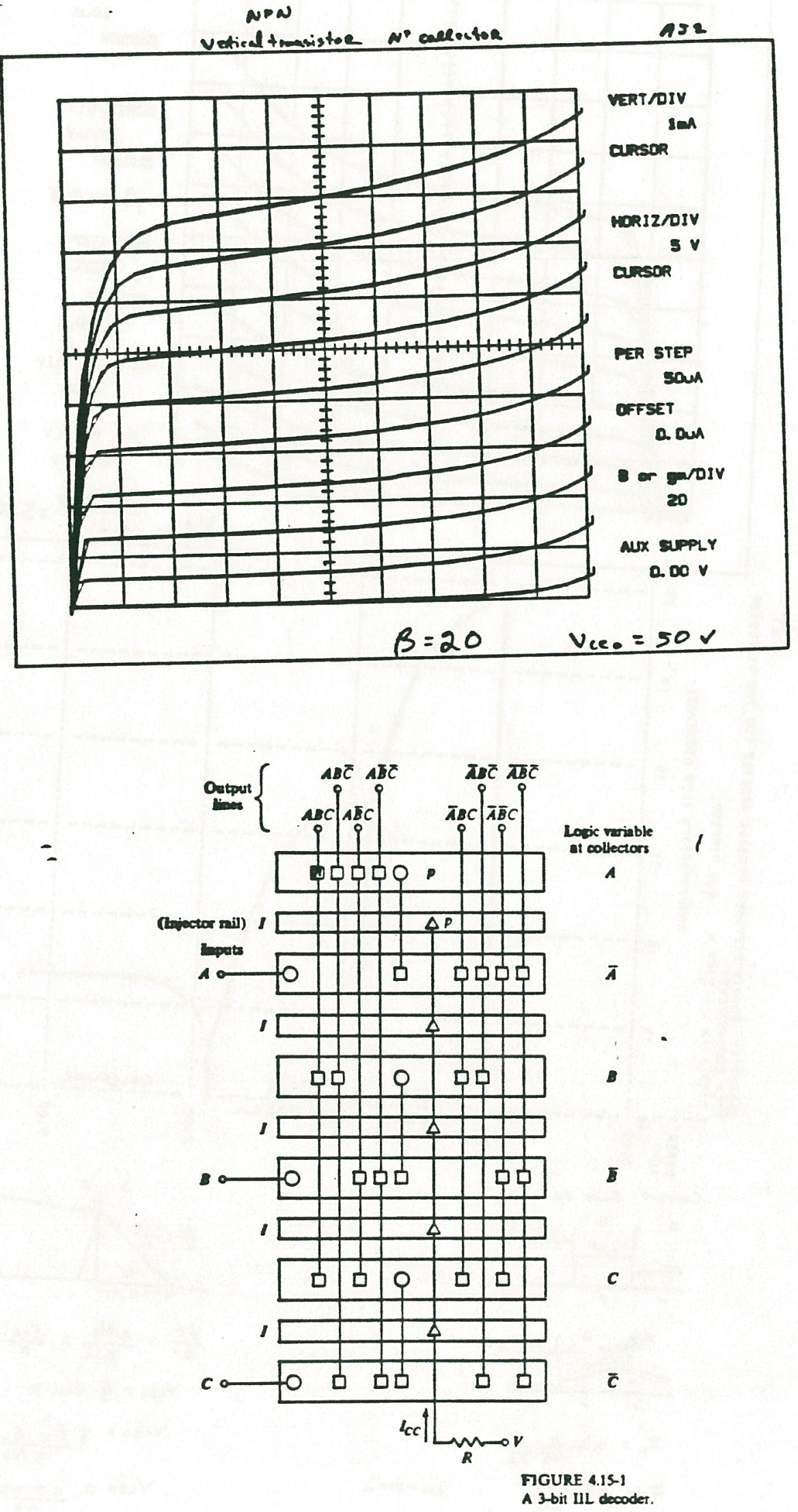